MELDUNGEN

TIA

\section{Erhöhtes Risiko auch für das Herz}

Eine transiente ischämische Attacke (TIA) ist nicht nur ein Alarmsignal für einen drohenden Schlaganfall, sie signalisiert auch ein erhöhtes Risiko für einen Myokardinfarkt. Im Durchschnitt liegt die jährliche Herzinfarktrate bei Patienten nach TIA bei etwa $1 \%$ und damit etwa doppelt so hoch wie in der Bevölkerung insgesamt, so das Ergebnis einer Studie. TIAPatienten unter 60 Jahren hatten im Vergleich zu ihren Altersgenossen sogar eine 15-mal so hohe Herzinfarktrate. Das absolute Risiko für einen Herzinfarkt nach TIA nahm erwartungsgemäß mit dem Alter zu und war bei Männern größer als bei Frauen.

Burns JD et al. Stroke 2011;

doi:10.1161/STROKEAHA.110.593723

\section{ADIPOSITAS}

\section{Dick vom Kirchgang?}

Der Mensch lebt nicht vom Brot allein - aber ganz ohne das leibliche Wohl geht es eben auch nicht. Das scheint für fleißige Kirchgänger ganz besonders zuzutreffen, wie die CARDIAStudie gezeigt hat: Danach sind Menschen, die als junge Erwachsene regelmäßig einen Gottesdienst besucht haben, signifikant häufiger in ihren mittleren Lebensjahren übergewichtig. In die Studie waren 2433 Männer und Frauen zwischen 20 und 32 Jahren aufgenommen und nach ihren religiösen Gewohnheiten befragt worden. Nach im Schnitt 18 Jahren wurde das kardiovaskuläre Risikoprofil der Probanden erfasst. Übergewicht und Adipositas waren bei den regelmäßigen Kirchgängern besonders häufig. Wer wenigstens einmal pro Woche zum Gottesdienst ging, hatte gegenüber den NichtKirchgängern ein um 50\% erhöhtes Risiko, übergewichtig zu werden.

Die Autoren wiesen allerdings darauf hin, dass bei Kirchgängern dafür andere Risikofaktoren wie Rauchen seltener sind und dass sie abgesehen von ihren Gewichtsproblemen in der Regel einen gesünderen Lebensstil aufweisen.

Feinstein MJ et al, Cardiovascular Disease Epidemiology and Prevention and Nutrition, Physical Activity and Metabolism 2011 Conference, Atlanta, 23.3.2011, Poster MP016

\section{Frühes Training bringt's Vorschulkinder als Ersthelfer überraschend kompetent}

\begin{abstract}
Iـ Inwieweit ist ein Fünfjähriger in der Lage, einen Notfall zu erkennen und erste Hilfe zu leisten? In einer schwedischen Studie stellten Kindergartenkinder nach einem Erste-HilfeKurs ihre Kompetenz in der Notsituation unter Beweis.

Die Forscher gingen davon aus, dass theoretisch jedes Kind bereits im Kindergartenalter mit einer Notsituation, bspw. einer bewusstlosen Erzieherin, konfrontiert sein kann; sie wollten herausfinden, ob ein Notfalltraining schon in diesem Alter sinnvoll ist. Dazu ließen sie insgesamt zehn Vier- bis Fünfjährige an einem Erste-Hilfe-Kurs teilnehmen und testeten zwei Monate nach Kursende deren Fähigkeiten in einem gestellten Unfallszenario. Die Mehrheit der Knirpse erwies sich als Ersthelfer überraschend kompetent: 70\% schätzten die Bewusstlosigkeit des fiktiven Unfallopfers richtig ein und hatten die Notrufnummer parat. $60 \%$ waren in der Lage, zu erkennen, ob das Opfer atmete, und 40\% erfüllten weitere Anforderungen wie Kenntnisse zur korrekten Lagerung und zum Frei-
\end{abstract}

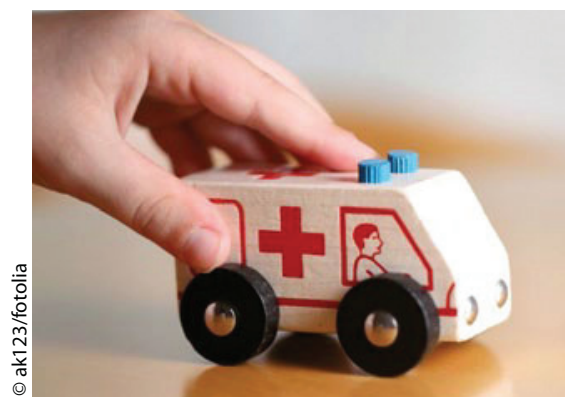

Tatütata: Knirps im Noteinsatz.

machen der Atemwege. Studienleiter Bollig sah sich bestätigt:„Erste-Hilfe-Training sollte spielerisch bereits im Kindergarten beginnen und im Schulalter verstärkt werden." Damit würden langfristig mehr Menschen ermutigt, im Ernstfall Erste Hilfe zu leisten.

EOI

Bollig G et al. Scandinavian Journal of Trauma, Resuscitation and Emergency Medicine 2011,19:13; doi:10.1186/1757-7241-19-13

\section{Yoga gegen Vorhofflimmern Mit dem „Sonnengruß" wieder im Rhythmus}

Dreimal pro Woche „Sonnengruß" und ein paar weitere Asanas, Atemübungen und Meditation, jedesmal für eine Dreiviertelstunde: das kann Flimmerepisoden fast zu halbieren. D. Lakkireddy, indischstämmiger Amerikaner von der Universität Kansas, untersuchte seinen Yoga-Ansatz zwar nur bei 49 Patienten mit paroxysmalem Vorhofflimmern; in dieser

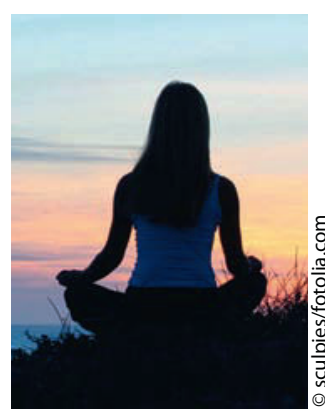

Mit Yoga in den Tag: So wird er hoffentlich flimmerfrei. ausreichen, um die Anzahl von

es im Schnitt 3,8mal; dagegen kam es während des Yogakurses, der ebenfalls über drei Monate lief, im Mittel nur zu 2,1 Episoden. $22 \%$ der Patienten wurden ihr $\varepsilon$ Leiden während der Kursphase komplett los. Zudem litten die Teilnehmer deutlich weniger unter Angst und Depression. Wenngleich er den Yogakursen einen erheblichen Einfluss auf die Frequenzregulation zusprach, warnte der Autor davor, sämtliche kleinen Gruppe war der Erfolg aber beachtlich. Innerhalb einer dreimonatigen Kontrollphase, in der die Teilnehmer ihr Routinesportprogramm absolvierten, fllimmerte"
Herzmedikamente abzusetzen; als Zusatzmaßnahme könnten die "Asanas" jedoch deutlich zu Buche schlagen.

EO II 